\title{
Constraints on the Intergalactic Magnetic Field from Gamma-Ray Observations of Blazars
}

\author{
J. D. Finke \\ Space Science Division Code 7653, US Naval Research Laboratory, 4555 Overlook Ave. SW, 20375, \\ USA \\ L. Reyes \\ Physics Department, California Polytechnic State University, San Luis Obispo, CA 94307, USA \\ M. Georganopoulos \\ Department of Physics, University of Maryland Baltimore County, 1000 Hilltop Circle, Baltimore, MD 21250, \\ USA and \\ NASA Goddard Space Flight Center, Code 660, Greenbelt, MD 20771, USA \\ on behalf of the Fermi-LAT Collaboration
}

\begin{abstract}
Gamma rays from distant blazars interact with the extragalactic background light, creating electron-positron pairs, and reducing the gamma-ray flux measured by ground-based atmospheric Cherenkov gamma-ray telescopes. These pairs can Compton-scatter the cosmic microwave background, creating a gamma-ray signature observable by the Fermi Large Area Telesope (LAT). The signature is also dependent on the intergalactic magnetic field (IGMF), since it can deflect the pairs from our line of sight, reducing the gamma-ray emission. We present preliminary constraints on the IGMF using Fermi-LAT and Cherenkov telescope observations, ruling out both very large and very small values of the IGMF strength.
\end{abstract}

\section{Introduction}

The extragalactic backgroung light (EBL) from the infrared (IR), through the optical and into the ultraviolet (UV) is dominated by emission, either directly or through dust absorption and reradiation, of all the stars which have ever existed in the observable universe. It contains information about the cosmological expansion, star formation history, dust extinction and radiation in the universe, and so can potentially provide constraints on a number of cosmologically interesting parameters. However, its direct detection is hampered by the bright foreground emission from the Earth's atmosphere, the solar system, and the Galaxy. This can be avoided by measurements from spacecraft outside the Earth's atmosphere [e.g., Bernstein 2007, Bernstein et al. 2002, Dwek et al. 1998, Hauser et al. 1998, Mattila 2003 or solar system Edelstein et al. 2000, Leinert et al. 1998, Matsuoka et al. 2011, Murthy et al. 2001, Toller 1983], or through galaxy counts, which in general give lower limits to the EBL [e.g., Madau \& Pozzetti 2000, Marsden et al. 2009]. See Hauser \& Dwek 2001] for a review of EBL measurements, constraints, and models.

Shortly after the discovery of the cosmic microwave background (CMB) radiation Penzias \& Wilson 1965], it was realized that this and other radiation fields would interact with extragalactic $\gamma$-rays, producing $e^{+} e^{-}$pairs, effectively absorbing the $\gamma$ rays Fazio \& Stecker 1970, Gould \& Schréder 1967]. In the 1990s, extragalactic high-energy $\gamma$-ray astronomy took two major leaps forward, one with the launch of the Compton Gamma-Ray Observatory and the first detections of extragalactic sources at high energies $(\mathrm{MeV}-\mathrm{GeV})$ by EGRET Hartman et al. 1992], and another at very-high energies (VHE; $\geq 0.1 \mathrm{TeV}$ ) by ground-based atmospheric Cherenkov telescopes [ACTs; e.g., Punch et al. 1992]. It was almost immediately realized that $\gamma$-ray observations of extragalactic blazars could be used to constrain the EBL Biller et al. 1995, Dwek \& Slavin 1994, Madau \& Phinnev 1996, Stecker \& de Jager 1993, Stecker et al. 1992]. The detection of the hard spectrum from the BL Lac 1ES 1101-232 with HESS seems to put strong constraints on the EBL, ruling out models that predict high opacity if one assumes the intrinsic VHE spectrum cannot be harder than $\Gamma=1.5$, where the differential photon flux $\Phi(E)=d N / d E \propto E^{-\Gamma}$ Aharonian et al. 2006]. However, the $\Gamma=1.5$ constraint has been questioned, and a number of theoretical possibilities have been raised that could account for harder VHE spectra Aharonian. Khangulvan. \& Costamante 2008, Böttcher, Dermer, \& Finke 2008, Stecker, Baring, \& Summerlin 2007]. Nonetheless, the basic idea, that the intrinsic VHE photon index cannot be harder than a certain number, has been used by many authors to constrain the EBL [e.g., Aharonian et al. 2007c, Albert et al. 2008, Finke \& Razzaque 2009, Mazin \& Raue 2007, Schroedter 2005].

The new era of $\gamma$-ray astronomy, began with the launch of the Fermi Gamma-Ray Space Telescope has brought additional constraints on the EBL. At the energies observed by the LAT, the extragalactic $\gamma$ ray sky below $\sim 10 \mathrm{GeV}$ is expected to be entirely transparent to $\gamma$-rays, at least back to the era of recombination $[z \sim 1000$; e.g., Finke et al. 2010, Oh 2001], while photons observed in the $10 \mathrm{GeV}-300$ 
GeV range should be attenuated by UV/optical photons if they originate from sources at $z \geq 0.5$. This suggests a possible way to constrain the EBL. The LAT spectrum below $10 \mathrm{GeV}$ can be extrapolated to higher energies, and should be an upper limit on the intrinsic spectrum in the range where the EBL attenuates the $\gamma$-rays. That is, everywhere for the $\gamma$ ray spectrum $d^{2} \log (\Phi) /(d \log (E))^{2} \leq 0$ (the spectrum is concave). This seems to be a reasonable assumption, since no convex $\gamma$-ray spectrum has been observed, and it is difficult to imagine theoretical ways to produce one, although see Section 5 for a discussion. Abdo et al. 2010] have used this technique to put upper limits on the optical/UV EBL absorption optical depth $\left(\tau_{\gamma \gamma}\right)$ for sources $z \geq 0.5$, and rule out with high significance some models which predict high $\tau_{\gamma \gamma}$ with data from the first 11 months of LAT operation from 5 blazars and 2 gamma-ray bursts (GRBs). More recently, Ackermann et al. 2012a used a similar technique in a composite fit to 150 BL Lacs with 46 months of LAT data to constrain the high- $z$ EBL even further, and found agreement with most recent models Domínguez et al. 2011, Finke et al. 2010, Franceschini et al. 2008, Gilmore et al. 2009, 2012, Kneiske \& Dole 2010].

Below $100 \mathrm{GeV}$, the universe is expected to be transparent out to $z \sim 0.1$, although VHE photons from this redshift range should be attenuated by interactions with IR EBL photons. Georganopoulos. Finke, \& Reves 2010 suggested a very similar technique to Abdo et al. [2010], applied to the VHE range. For those sources detected by both LAT and an ACT, one can extrapolate the LAT spectrum (which should be unattenuated for these sources at low $z$ ) into the VHE range, and use it as an upper limit on the intrinsic VHE flux. As with the LAT-only case, a comparison of this upper limit with the observed VHE flux allows one to compute an upper limit on $\tau_{\gamma \gamma}$. Georganopoulos et al. 2010] used this technique to show that models which predicted high $\tau_{\gamma \gamma}$ in the VHE range were strongly disfavored. Another possible way to estimate the intrinsic spectrum of a source, and thus estimate $\tau_{\gamma \gamma}$, comes from modeling the full radio to $\mathrm{GeV} \gamma$-ray spectral energy distribution (SED) of a $\gamma$-ray blazar with a standard synchrotron/synchrotron self-Compton (SSC) model. The SSC spectrum can be extrapolated to the VHE regime, and compared with observations to estimate $\tau_{\gamma \gamma}$ Mankuzhivil et al. 2011. Dominguez et al. 2013. has applied this technique to a sample of $\sim 15$ LAT and ACT-detected blazars to constrain the cosmic $\gamma$-ray horizon, i.e., the energy where $\tau_{\gamma \gamma}=1$ for a certain redshift. Dwek \& Krennrich [2012] present a comprehensive review of recent attempts to constrain the EBL with $\gamma$-ray observations.

These constraints come with caveats, however. One possibility is that the electron positron pairs that are produced by the $\gamma$-ray-EBL photon in- teractions Compton-scatter the CMB photons, producing $\mathrm{GeV} \gamma$-ray emission which could themselves be absorbed by interactions with the EBL, producing a cascade Aharonian et al. 1994, Dai et al. 2002, Plaga 1995, ?]. If the intergalactic magnetic field (IGMF) strength is low, the pairs will not be significantly deflected from our line of sight, and this could produce an observable feature in the LAT bandpass [e.g., Neronov \& Semikoz 2009]. Indeed, recently several authors have even used the non-detection of these cascades to put lower limits on the IGMF strength [e.g., Dolag et al. 2011, Neronov \& Vovk 2010, Tavecchio et al. 2010]. In general these efforts depend on the fact that emission from $\mathrm{TeV}$ blazars is relatively constant over long periods of time. $\mathrm{TeV}$ variability has not been observed in some blazars, [e.g., Aharonian et al. 2006, 2007c], although many blazars are highly variable at these energies [e.g., Aharonian et al. 2007a]. Observing and studying the time-dependent EBL-induced pair cascades from GRBs and blazar flares has been suggested as a way to probe the IGMF parameters Ichiki et al. 2008, Murase et al. 2008, Razzaque et al. 2004]. The possibility of variable $\mathrm{TeV}$ emission has led to caveats in interpreting the IGMF constraints from apparently non-variable $\mathrm{TeV}$ blazars Dermer et al. 2011, Tavlor et al. 2011]. Other uncertainties such as the EBL intensity and $\mathrm{TeV}$ spectra errors, blazar jet geometry and Doppler factor, can further decrease the lower limits on the IGMF [Arlen et al. 2012].

In this paper we report on our efforts to constrain both the EBL absorption optical depth, $\tau_{\gamma \gamma}$ and the IGMF strength $\left(B_{I G}\right)$ and coherence length $\left(\lambda_{B}\right)$ using $\gamma$-ray observations of the blazars 1ES 1101-232 and 1ES 0229+200 from both LAT and ACTs. This technique can be seen as an extension of previous work by Georganopoulos, Finke, \& Reyes 2010]. We make use of data from the first 3.5 years of LAT operation, the analysis of which is described in Section 2 and $\mathrm{ACT}$ spectra from the literature. Our technique for constraining the IGMF is described in Section 3 , We report our preliminary constrains on the IGMF parameters in Section 4. Finally we discuss these results, their caveats and implications in Section [5.

\section{LAT Analysis}

To determine the LAT spectra of 1ES 1101-232 and 1ES $0229+200$ we considered all LAT events collected since the start of the mission for 3.5 years of operation (i.e. until 2012 February 16), a significant increase in statistics with respect to previous efforts. The data were analyzed using an official release of the Fermi ScienceTools (v9r27) and Pass 7 instrument response functions. Only photons satisfying the CLEAN event selection are included in the analysis in order to reduce the likelihood that charged-particle background 
particles are mistaken as high-energy photons. As usual, the spectral analysis of each source is based on the maximum likelihood technique using the standard likelihood analysis software. In both cases we considered all the point-like sources within $15^{\circ}$ from the source position (as determined from the 2FGL catalog and improved diffuse background components (Galactic and extragalactic). A likelihood ratio test was used to find the best spectral model (power-law, broken power law, and log parabola) that fits the data. In both cases, there was insufficient evidence for deviation from a power-law spectrum.

In addition to the standard maximum likelihood analysis performed to find the best fit to the data, the Log-of-the-likelihood $(L L)$ profile as a function of the source's flux normalization $\left(F_{0}\right)$ was calculated in order to fully characterize the uncertainty on this parameter. Thus, instead of assuming a perfect Gausian distribution for the error of $F_{0}$ and using 1,2 , and 3 standard deviations to calculate the $68 \%, 95 \%$ and $99 \%$ confidence intervals, we used the $L L$ profile to calculate the actual confidence intervals assuming that $-2 \Delta(L L)$ is distributed as the chi-square probability distribution with one degree of freedom. We found that this approach is necessary in order to correctly determine the flux probability distribution of weak LAT sources such as $1 \mathrm{ES} 0229+200$ and $1 \mathrm{ES}$ $1101-232$.

\section{Method for Constraining Models}

Our technique for constraining the EBL and IGMF is based on Georganopoulos et al. [2010], with extentions including a sophisticated Monte Carlo (MC) technique to accurately determine the significance of the constraints, and the addition of a pair cascade component.

\subsection{Technique Assumptions}

We make the following assumptions, the first three of which are idential to the ones of Georganopoulos et al. [2010]:

\#1 We assume the MeV-TeV flux from BL Lacs in our sample are produced cospatially from the sources themselves (and not UHECR interactions in intergalactic space), and that they are never convex (i.e., never is $\left.d^{2} \log (\Phi) /(d \log (E))^{2}>0\right)$.

\#2 We assume the objects are not variable at $\gamma$ rays within the statistical uncertainties of the measurements. Indeed, we have selected sources for our sample for which $\gamma$-ray variability has not been reported, in either the LAT or ACTs.

\#3 We assume the $\gamma$ rays will not avoid being absorbed on their path to Earth, by converting to axions or some other exotic mechanism [e.g., de Angelis et al. 2007, Sánchez-Conde et al. 2009

\#4 We assume pairs created by $\gamma$-ray-EBL photon interactions will Compton-scatter the CMB, and will lose energy primarily through scattering and not through intergalactic plasma beam instabilities Broderick et al. 2012, Schlickeiser et al. 2012a,b]. Our technique for including the cascade component is similar to the one used by Mever et al. 2012].

We discuss the viability of these assumptions in Section 5 .

\subsection{Ruling out Models}

Our technique for ruling out a model is illustrated in Figure 1 and has the following steps:

Step 1. Select the model we wish to rule out. This includes selecting an EBL model (taken from the literature), selecting an IGMF strength $\left(B_{I G}\right)$ and coherence length $\left(\lambda_{B}\right)$; and selecting a blazar jet radiation opening angle and a "blazar lifetime" ( $\left.t_{\text {blazar }}\right)$, that is, the length of time the blazar has been emitting $\gamma$-rays with its current luminosity Dermer et al. 2011].

Step 2. Given the integrated LAT flux $\left(F_{L A T}\right)$ and photon index $(\Gamma)$ and their errors for a particular blazar (see Section 2), we draw a random $F_{L A T}$ and $\Gamma$ from a probability distribution function (PDF) which represents their errors.

Step 3. For each energy bin of the VHE spectrum, with a measured flux and error, we draw a random flux, $F_{V H E}$, assuming the flux errors are distributed as a normal distribution. Each randomly drawn $F_{V H E}(E)$ is deabsorbed with the EBL model we are testing to give an intrinsic flux, $F_{V H E \text {,int }}(E)=$ $\exp \left(\tau_{\gamma \gamma}(E)\right) \times F_{V H E}(E)$.

Step 4. From $F_{V H E \text {,int }}(E)$, the contribution of the $e^{+} e^{-}$pairs Compton-scattering the CMB, $F_{\text {cascade }}$ is calculated. The deabsorbed emission is assumed not to extend beyond the highest energy bin, the most conservative assumption we can make. The cascade flux $F_{\text {cascade }}$ is calculated using the formula of Dermer et al. 2011] and Dermer 2012]. The minor corrections to this formula from Meyer et al. 2012. should have no real effect on the results, since they affect the lowest flux portion of the cascade spectrum.

Step 5. The randomly drawn LAT power-law spectrum from step 2 is extrapolated to the VHE regime. For this MC iteration, the model is considered rejected if one of two criteria are met: (i) the cascade flux $F_{\text {cascade }}$ exceeds the randomly drawn LAT integrated flux $F_{L A T}$; or (ii) any one of the deabsorbed flux bins from $F_{V H E \text {,int }}(E)$ exceed the extrapolated LAT flux, $F_{L A T, \text { ext }}$, unless $0.01 F_{L A T} \leq F_{\text {cascade }}<F_{L A T}$, in which case the model is never rejected for this iteration. If the cascade flux makes up a significant fraction of the observed LAT flux, we do not believe it can be extrapolated to the VHE regime and used to 


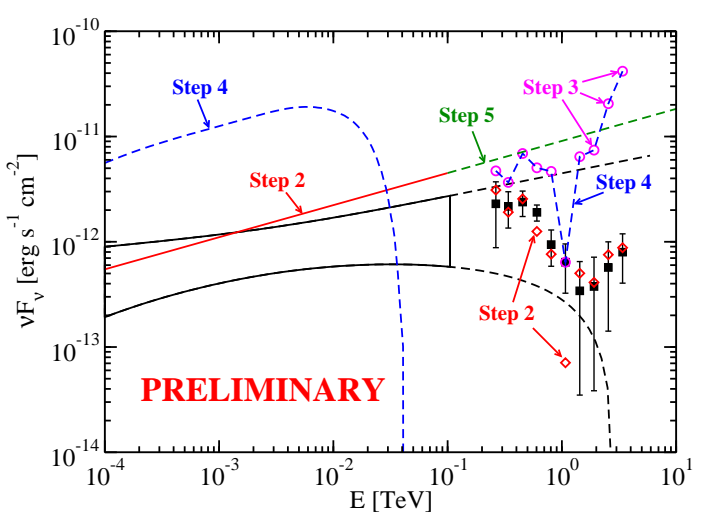

Figure 1: This figure illustrates many of the steps in our method for ruling out models from Section 3.2 using the $\gamma$-ray spectrum for $1 \mathrm{ES} 1101-232$. The LAT spectrum is shown as the bowtie, along with this spectrum extrapolated to the VHE regime as the dashed curves. The observed HESS spectrum is shown as the filled squares. The randomly drawn HESS points shown as empty diamonds $\left(F_{V H E}\right)$ and the randomly drawn LAT spectrum is shown as a line, both of which are labeled "Step 2". The deabsorbed points are shown as the circles $\left(F_{V H E, \text { int }}\right)$ and labeled "Step 3". The cascaded component and the interpolated VHE spectrum used to calculate it are shown as dashed curves labeled "Step 4". The LAT spectrum extrapolated into the VHE regime is shown as the dashed line labeled "Step 5". For the MC iteration shown here, the model is ruled out by both criteria in Step 5, since $F_{L A T}<F_{\text {cascade }}$ and for several points $F_{L A T, \text { ext }}<F_{V H E \text {,int }}$.

constrain models. The rejection criteria in this step are based on ones from Georganopoulos et al. 2010. and Mever et al. 2012.

Step 6. Steps $2-5$ above are repeated $N_{\text {trials }}$ times (we use $N_{\text {trials }}=10^{6}$ ) and the number of times the model is rejected $N_{\text {reject }}$ is counted. The probability the model is ruled out is $P_{\text {reject }}=N_{\text {reject }} / N_{\text {trials }}$.

\section{Constraints on IGMF}

We apply the method outlined in Section 3.2 to $\gamma$ ray observations of 1ES $0229+200$ and 1ES 1101-232. We use the HESS spectra for these sources from Aharonian et al. 2007c and Aharonian et al. 2007b], respectively, and the LAT spectra calculated as outlined in Section 2. We used the EBL model from Finke et al. [2010], a jet radiation opening angle of 0.1 rad, and a blazar lifetime of $t_{\text {blazar }}=H_{0}^{-1}$, where $H_{0}$ is the Hubble constant; that is, we assume the blazar has been emitting for essentially the entire age of the universe. Neither of these objects have been shown to be variable at $\gamma$-ray energies, with either the LAT or $\mathrm{TeV}$ instruments.

The results from the application to 1ES $0229+200$

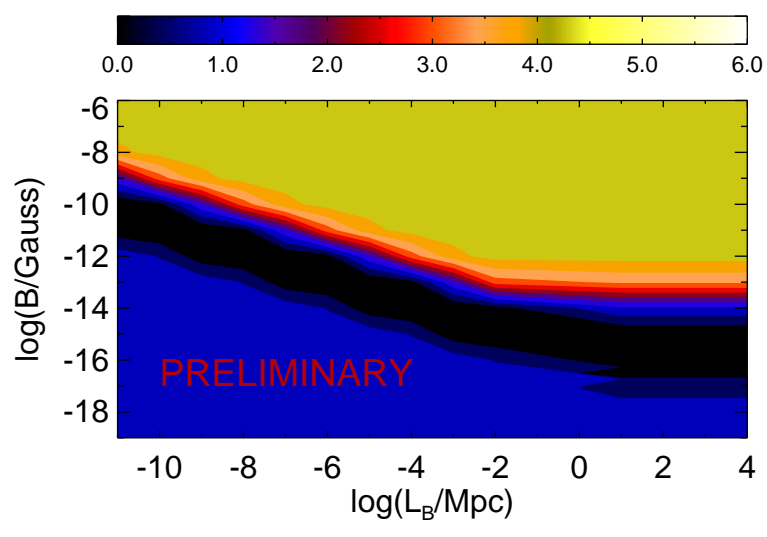

Figure 2: The values of parameter space of $B$ and $L_{B}$ which are ruled out by our method, using LAT and HESS observations of 1ES $0229+200$. The significance that the values are ruled out are given by the colors, in number of sigma, as indicated by the color bar.

and 1ES 1101-232 can be found in Figs. 2 and 3 , respectively. The probabilities that values of parameter space are ruled are converted to number of sigma, assuming the errors are distributed normally. High values of $B_{I G}$ are ruled out by the constraint that deabsorbed TeV flux cannot exceed the LAT spectrum spectrum extrapolated into this regime; and low values of $B$ are ruled out by the constraint that the cascade cannot exceed LAT flux (see Section 3.2. step 5). A black strip in these figures indicates allowed values of $B_{I G}$ and $L_{B}$. Here the cascade flux was less than the observed LAT flux, but $>0.01$ of the LAT flux, so that the LAT flux could not be simply extrapolated into the $\mathrm{TeV}$ regime.

The combined results for both $1 \mathrm{ES} 0229+200$ and 1ES 1101-232 are shown in Fig. 4. High values of $B_{I G}$ are ruled out at greater than $5 \sigma$, while lower values are ruled out at greater than $3 \sigma$.

\section{Discussion}

We have used LAT and $\mathrm{TeV}$ data to constrain IGMF parameters. Our preliminary results indicate high values of $B_{I G}$ are ruled out with $>5 \sigma$ significance. This means that either one of our model assumptions is not correct (Section 3.1), one of our parameters is not correct, or the cascade flux must contribute at least $1 \%$ to the emission observed by the LAT. We discuss these possibilities below.

If the $\mathrm{GeV}$ and $\mathrm{TeV} \gamma$-rays are not produced co-spatially (model assumption \#1), this could explain our results at high $B_{I G}$. It is possible they are not Böttcher et al. 2008, Essev et al. 2011, 2010, Essey \& Kusenko 2010]. In this case one might ex- 


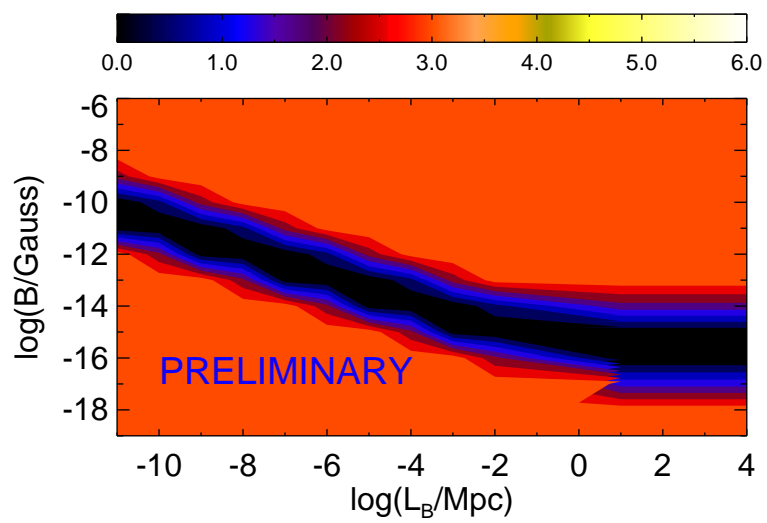

Figure 3: Similar to Fig. 2] only for 1ES 1101-232.

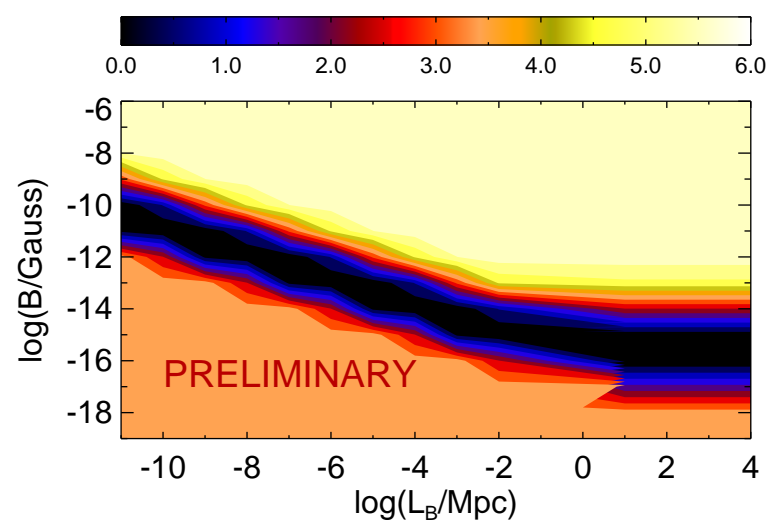

Figure 4: Similar to Figs. 2 and 3, only here we show the combined results for both 1ES 1101-232 and 1ES $0229+200$.

pect the $\mathrm{TeV}$ flux to be larger than the extrapolated LAT flux. If the two sources we used are variable (assumption \#2), the variablity would have to be greater than the observational errors, which are quite large, to invalidate our results. Our results could be interpreted as evidence for axion-like particles (assumption \#3), which would allow TeV photons to avoid much of the EBL attenuation. This would be difficult to distinguish, although with sufficiently accurate observations certain $\gamma$-ray signatures may be found Sánchez-Conde et al. 2009, e.g.,]. Finally, if all our other assumptions are correct, the cascade component is likely to exist at the $>5 \sigma$ level. This implies that it is very unlikely that plasma beam instabilities would eliminate the cascade Broderick et al.|2012, assumption \#4]. See also Venters \& Pavlidou [2012] for a critical assesment of this possibility.

Of our model parameters, the EBL model of Finke et al. 2010] is unlikely to be wrong to a large degree, given its proximity to galaxy counts and agreement with other models. The timescale the blazar has been operating would not effect the upper limits on $B_{I G}$, nor could the jet opening angle. We will explore other EBL models in future work.

The final possibility is that there is indeed a significant cascade component created, and the upper limits on $B_{I G}$ and $L_{B}$ can be taken at face value. We note that in this case, the parameters are in the range where one might expect extended $\mathrm{GeV}$ emission (" $\gamma$-ray halos") around TeV sources to be detectable by the LAT [Neronov \& Semikoz 2009]. The detection of this emission or lack thereof could provide confirmation of these parameter constraints, or the other possibilities discussed above. The detection of these halos in the Fermi era has been controversial Ackermann et al. 2013, Ando \& Kusenko 2010, Neronov et al. 2011].

The constraints on lower magnetic field values from the cascade are less certain, not just because of their lower significance but also due to their dependence on parameter assumptions. The timescale the blazar has been emitting $\mathrm{TeV} \gamma$-rays or changes the opening angle can significantly effect the cascade Arlen et al. 2012, Dermer et al. 2011]. We will explore how changes of these parameters effect our results.

The IGMF may have been generated in phase transitions in the early universe [e.g., Grasso \& Rubinstein 2001]. Our preliminary results ruling out low $B_{I G}$ disfavor IGMFs generated from electro-weak phase transitions, if the generated fields are non-helical Neronov \& Semikoz 2009]. However, as discussed above, the lower magnetic field values are dependent on caveats including those regarding source variability. If the IGMF is generated from phase transitions, and if it is possible to constrain $B_{I G} \geq 10^{-15} \mathrm{G}$, then it will be almost impossible to detect gravitational waves from inflation [Fujita \& Mukohyama 2012]. The IGMF parameters also have implications for the diffuse gamma ray background and its anisotropy Ackermann et al. 2012b, Venters \& Pavlidou 2012].

\section{Acknowledgments}

The Fermi LAT Collaboration acknowledges support from a number of agencies and institutes for both development and the operation of the LAT as well as scientific data analysis. These include NASA and DOE in the United States, CEA/Irfu and IN2P3/CNRS in France, ASI and INFN in Italy, MEXT, KEK, and JAXA in Japan, and the K. A. Wallenberg Foundation, the Swedish Research Council and the National Space Board in Sweden. Additional support from INAF in Italy and CNES in France for science analysis during the operations phase is also gratefully acknowledged. 


\section{References}

Abdo, A. A., et al. 2010, ApJ, 723, 1082

Ackermann, M., et al. 2012a, Science

—. 2012b, Phys. Rev. D, 85, 083007

—. 2013, ApJ, 765, 54

Aharonian, F., et al. 2006, Nature, 440, 1018

—. 2007a, ApJL, 664, L71

-. 2007b, A\&A, 470, 475

-. 2007c, A\&A, 475, L9

Aharonian, F. A., Coppi, P. S., \& Voelk, H. J. 1994, ApJL, 423, L5

Aharonian, F. A., Khangulyan, D., \& Costamante, L. 2008, MNRAS, 387, 1206

Albert, J., et al. 2008, Science, 320, 1752

Ando, S., \& Kusenko, A. 2010, ApJL, 722, L39

Arlen, T. C., Vassiliev, V. V., Weisgarber, T., Wakely,

S. P., \& Yusef Shafi, S. 2012, ArXiv e-prints

Bernstein, R. A. 2007, ApJ, 666, 663

Bernstein, R. A., Freedman, W. L., \& Madore, B. F. 2002, ApJ, 571, 56

Biller, S. D., et al. 1995, ApJ, 445, 227

Böttcher, M., Dermer, C. D., \& Finke, J. D. 2008, ApJL, 679, L9

Broderick, A. E., Chang, P., \& Pfrommer, C. 2012, ApJ, 752, 22

Dai, Z. G., Zhang, B., Gou, L. J., Mészáros, P., \& Waxman, E. 2002, ApJL, 580, L7

de Angelis, A., Roncadelli, M., \& Mansutti, O. 2007, Phys. Rev. D, 76, 121301

Dermer, C. D. 2012, ArXiv:1202.2814

Dermer, C. D., Cavadini, M., Razzaque, S., Finke, J. D., Chiang, J., \& Lott, B. 2011, ApJL, 733, L21

Dolag, K., Kachelriess, M., Ostapchenko, S., \& Tomàs, R. 2011, ApJL, 727, L4

Domínguez, A., et al. 2011, MNRAS, 410, 2556

Dominguez, A., et al. 2013, ApJ, submitted

Dwek, E., \& Krennrich, F. 2012, ArXiv e-prints

Dwek, E., \& Slavin, J. 1994, ApJ, 436, 696

Dwek, E., et al. 1998, ApJ, 508, 106

Edelstein, J., Bowyer, S., \& Lampton, M. 2000, ApJ, 539, 187

Essey, W., Kalashev, O., Kusenko, A., \& Beacom, J. F. 2011, ApJ, 731, 51

Essey, W., Kalashev, O. E., Kusenko, A., \& Beacom, J. F. 2010, Physical Review Letters, 104, 141102

Essey, W., \& Kusenko, A. 2010, Astroparticle Physics, 33,81

Fazio, G. G., \& Stecker, F. W. 1970, Nature, 226, 135

Finke, J. D., \& Razzaque, S. 2009, ApJ, 698, 1761

Finke, J. D., Razzaque, S., \& Dermer, C. D. 2010, ApJ, 712, 238

Franceschini, A., Rodighiero, G., \& Vaccari, M. 2008, A\&A, 487, 837

Fujita, T., \& Mukohyama, S. 2012, JCAP, 10, 34

Georganopoulos, M., Finke, J. D., \& Reyes, L. C. 2010, ApJL, 714, L157
Gilmore, R. C., Madau, P., Primack, J. R., Somerville, R. S., \& Haardt, F. 2009, MNRAS, 399, 1694

Gilmore, R. C., Somerville, R. S., Primack, J. R., \& Domínguez, A. 2012, MNRAS, 422, 3189

Gould, R. J., \& Schréder, G. P. 1967, Physical Review, 155,1408

Grasso, D., \& Rubinstein, H. R. 2001, Physics Reports, 348, 163

Hartman, R. C., et al. 1992, ApJL, 385, L1

Hauser, M. G., \& Dwek, E. 2001, ARA\&A, 39, 249

Hauser, M. G., et al. 1998, ApJ, 508, 25

Ichiki, K., Inoue, S., \& Takahashi, K. 2008, ApJ, 682, 127

Kneiske, T. M., \& Dole, H. 2010, A\&A, 515, A19

Leinert, C., et al. 1998, A\&AS, 127, 1

Madau, P., \& Phinney, E. S. 1996, ApJ, 456, 124

Madau, P., \& Pozzetti, L. 2000, MNRAS, 312, L9

Mankuzhiyil, N., Ansoldi, S., Persic, M., \& Tavecchio, F. 2011, ApJ, 733, 14

Marsden, G., et al. 2009, ApJ, 707, 1729

Matsuoka, Y., Ienaka, N., Kawara, K., \& Oyabu, S. 2011, ApJ, 736, 119

Mattila, K. 2003, ApJ, 591, 119

Mazin, D., \& Raue, M. 2007, A\&A, 471, 439

Meyer, M., Raue, M., Mazin, D., \& Horns, D. 2012, A\&A, 542, A59

Murase, K., Takahashi, K., Inoue, S., Ichiki, K., \& Nagataki, S. 2008, ApJL, 686, L67

Murthy, J., Henry, R. C., Shelton, R. L., \& Holberg, J. B. 2001, ApJL, 557, L47

Neronov, A., \& Semikoz, D. V. 2009, Phys. Rev. D, 80,123012

Neronov, A., Semikoz, D. V., Tinyakov, P. G., \& Tkachev, I. I. 2011, A\&A, 526, A90

Neronov, A., \& Vovk, I. 2010, Science, 328, 73

Oh, S. P. 2001, ApJ, 553, 25

Penzias, A. A., \& Wilson, R. W. 1965, ApJ, 142, 419

Plaga, R. 1995, Nature, 374, 430

Punch, M., et al. 1992, Nature, 358, 477

Razzaque, S., Mészáros, P., \& Zhang, B. 2004, ApJ, 613, 1072

Sánchez-Conde, M. A., Paneque, D., Bloom, E., Prada, F., \& Domínguez, A. 2009, Phys. Rev. D, 79,123511

Schlickeiser, R., Elyiv, A., Ibscher, D., \& Miniati, F. 2012a, ApJ, 758, 101

Schlickeiser, R., Ibscher, D., \& Supsar, M. 2012b, ApJ, 758,102

Schroedter, M. 2005, ApJ, 628, 617

Stecker, F. W., Baring, M. G., \& Summerlin, E. J. 2007, ApJL, 667, L29

Stecker, F. W., \& de Jager, O. C. 1993, ApJL, 415, L71+

Stecker, F. W., de Jager, O. C., \& Salamon, M. H. 1992, ApJL, 390, L49

Tavecchio, F., Ghisellini, G., Foschini, L., Bonnoli, G., Ghirlanda, G., \& Coppi, P. 2010, MNRAS, 406, L70 
Taylor, A. M., Vovk, I., \& Neronov, A. 2011, A\&A, Venters, T. M., \& Pavlidou, V. 2012, ArXiv:1201.4405 529, A 144

Toller, G. N. 1983, ApJL, 266, L79 